\title{
The Association between Cardiovascular Risk and Elevated Triglycerides
}

\author{
Djanggan Sargowo*, Olivia Handayani \\ Department of Cardiology and Vascular Medicine, Faculty of Medicine Brawijaya University - Dr. Saiful Anwar Hospital, Jl. Veteran, \\ Malang, Indonesia \\ *Corresponding author. E-mail: djanggan@yahoo.com
}

Received date: Nov 3, 2015; Revised date: Sep 30, 2016; Accepted date: Nov 16, 2016

\section{Abstract}

B ACKGROUND: The association between elevated triglycerides and cardiovascular risk has been extensively studied. The elevated level of triglycerides occurs through abnormalities in hepatic very low-density lipoprotein (VLDL) production and intestinal chylomicron synthesis, dysfunctional lipoprotein lipase (LPL)-mediated lipolysis or impaired remnant clearance.

CONTENT: Hypertriglyceridemia (HTG) commonly leads to a reduction in high-density lipoprotein (HDL) and increase in atherogenic small dense low-density lipoprotein (LDL) cholesterol, called the atherogenic dyslipidemia (AD). Triglycerides may also stimulate atherogenesis by mechanisms, such excessive release of free fatty acids, and production of pro-inflammatory cytokines, fibrinogen, coagulation factors and impairment of fibrinolysis. Genetic

\section{Introduction}

Atherosclerosis is the main process of abnormal lipoprotein. Prospective epidemiological studies consistently demonstrate the elevated plasma low-density lipoprotein cholesterol (LDL-C) is associated with increased risk of cardiovascular disease (CVD). However, it may be partly due to atherogenic dyslipidemia (AD), which clusters with hypertension, central obesity and insulin resistance, collectively known as metabolic syndrome (MetS).(1) $\mathrm{AD}$ is characterized by high plasma triglycerides, low high-density lipoprotein cholesterol (HDL-C) and a studies strongly support hypertriglyceridemia (HTG) and high concentration of triglyceride-rich lipoprotein (TRL) as causal risk factors for cardiovascular disease. Therefore, lipid management is crucial in reducing cardiovascular risk. Combination of lipid lowering drug therapy may be needed to achieve both LDL and non-HDL cholesterols treatment goal for cardiovascular disease prevention in patients with elevated triglyceride levels, particularly those with triglyceride $\geq 500 \mathrm{mg} / \mathrm{dL}$.

SUMMARY: LDL and non-HDL cholesterol can be a promising target therapy in HTG. Additional clinical outcomes data are needed to provide a more evidencebased rationale for clinical lipid management of hypertriglyceridemic patients.

KEYWORDS: hypertriglyceridemia, non-HDL cholesterol, dyslipidemia, CV risk

Indones Biomed J. 2017; 9(1): 17-22 high concentration of apolipoprotein (apo)-B-containing lipoproteins, particularly elevated small, dense LDL particles. The LDL particles in AD are smaller and denser, and have an increased atherogenic potential; small, dense HDL particles also occur. Based on Dyslipidemia International Study conducted on 22063 statin-treated outpatients (with or without diabetes) in Canada and Europe, it was found that these patients had persistent elevated triglycerides and/ or low HDL-C levels. Besides, more than $85 \%$ of Caucasian adults (age $>35$ years) with a mild-to-moderate elevation in plasma triglyceride and low HDL-C phenotype have the MetS.(1,2) Lipid profile in AD is shown in Table 1. Integral to $\mathrm{AD}$ is a dysregulation of very low-density 
Table 1. Lipid profile in AD.(2) (with modifications)

\begin{tabular}{lcc}
\hline \multicolumn{1}{c}{ Parameter } & Atherogenic dyslipide mia & Normal value \\
\hline LDL-C & $\begin{array}{c}\text { Normal/slightly increase in LDL-C levels } \\
\text { Small dense LDL levels increase }\end{array}$ & $\mathrm{n} / \mathrm{a}$ \\
& & \\
HDL-C & HDL-C levels decrease & Men $<40 \mathrm{mg} / \mathrm{dL}(<1.0 \mathrm{mmol} / \mathrm{L})$ \\
& Small dense HDL levels increase & Women $<50 \mathrm{mg} / \mathrm{dL}(<1.3 \mathrm{mmol} / \mathrm{L})$ \\
Triglycerides & Total triglycerides levels increase & \\
& VLDL triglycerides levels increase & Women $<50 \mathrm{mg} / \mathrm{dL}(<1.3 \mathrm{mmol} / \mathrm{L})$ \\
\hline
\end{tabular}

lipoprotein (VLDL) metabolism. It is caused by hepatic insulin resistance related to anomalous fat accumulation in visceral adipose tissue and liver. Oversecretion of VLDL and chylomicrons by the liver and intestine, coupled with decreased catabolism, raises the plasma pool of triglyceride-rich lipoproteins (TRLs) including remnant lipoproteins. Increased heteroexchange of neutral lipids between TRLs and LDLs and HDLs via cholesteryl ester transfer protein (CETP) results in remodeling of LDLs and HDLs to form correspondingly smaller, denser particles. Lipoprotein lipase (LPL) activity is decreased in skeletal muscle and adipose tissue owing to the inhibitory effects of insulin resistance. VLDL overproduction occurs in insulin resistant humans and in patients with type 2 diabetes mellitus. $(1,3)$ Complex genetic factor also plays a role in $\mathrm{AD}$ that is in the interaction between multiple genetic variants that perturb lipoprotein metabolism. Based on family-based and twin studies, approximately $50 \%$ of the total variance in plasma triglyceride, HDL-C and small dense LDL levels are attributed to genetic factors. Patients with familial combined hyperlipidemia (FCHL) commonly have a predominance of small dense LDL particles and elevated apo-B-100 levels. Genetic polymorphisms' in CETP, LPL, apo-A4, lipase C(LIPC), polypeptideN-acetylegalactosaminyltransferase 2 (GALNT2) and max-like protein $\mathrm{X}$ (MLX) interacting protein like (MLXIPL) are strongly associated with this atherogenic lipoprotein phenotype and cardiovascular risk.

Effective diagnosis and management of $\mathrm{AD}$ requires an overall for CVD risk, including age, presence of established CVD, hypertension or diabetes mellitus, family history of CVD and smoking.(1) A recent review proposed that nonHDL-C was used to provide a better measure of $\mathrm{CV}$ risk than LDL-C levels.(2)

\section{Hypertriglyceridemia}

HTG refers to an elevation of fasting triglyceride levels above normal ( $>150 \mathrm{mg} / \mathrm{dL})$. The sharp rise in the incidence of obesity was in line with that increasing prevalence during the past few decades. In 2010, there were 74.6 million US adults have elevated triglyceride levels, with 36.4 million of them had high triglyceride levels (200 to $499 \mathrm{mg} / \mathrm{dL}$ ).(4)

HTG may be categorized as borderline high (150-199 $\mathrm{mg} / \mathrm{dL}$ ), high (200-499 mg/dL), and very high (above 500 $\mathrm{mg} / \mathrm{dL}$ ). The common causes for HTG are familial LPL deficiency, chylomicronemia, elevated levels of VLDL, reduced levels of HDL, also the presence of type 1 and 2 diabetes mellitus. HTG itself can be divided into primary and secondary types, but a molecular basis for primary HTG has been found in less than $5 \%$ of cases and for secondary cases, no genetic susceptibility component that is reproducible. $(4,5)$

Primary HTG is mainly due to genetic predisposition. Chylomicrons normally are cleared rapidly from plasma by LPL with apo-C-II as a cofactor. Familial chylomicronemia (hyperlipoproteinemia type I, in the Fredrickson system) and primary mixed hyperlipidemia (type 5) are each characterized by the pathologic presence of chylomicrons after a 12-14-hour fasting period. Familial hypertriglyceridemia (FHTG)/hyperlipoproteinemia type 4 is interpreted by an isolated elevation of VLDL, which is not as triglyceride-rich as chylomicrons are. This familial disorder has a population prevalence of some 5 to $10 \%$ and is associated with increased risk of CVD, obesity, insulin resistance, diabetes, hypertension and hyperuricemia. Moderately elevated plasma triglyceride (2.8-10 $\mathrm{mmol} / \mathrm{L})$ is commonly found with low levels of HDL-C in those patients. 
The inheritance pattern of familial combined hyperlipoproteinemia (FCHL, type 2B) is one of an autosomal dominant with a population prevalence of $2-5 \%$. It is characterized by multiple lipoprotein abnormalities due to hepatic overproduction of apoB-containing VLDL, intermediate-density lipoprotein (IDL) and LDL. Familial dysbetalipoproteinemia (type 3 ) has a population prevalence of 1-2 in 20,000 and is due to the accumulation of cholesterol-rich VLDL, which results in a higher ratio of core cholesteryl ester to triglyceride $(>0.3)$ than in normal VLDL (0.2). This abnormality is homozygotic type for the binding defective apo-E E2 isoform. $(4,6)$

In secondary HTG, there is some metabolic conditions that are frequently associated with high triglyceridemia levels. Obesity with poorly controlled type 2 diabetes is the most common of metabolic stressor. HTG is also associated with alcohol consumption; this is mainly the result of increased plasma VLDL. Elevated LDL-C is commonly found in patients with renal disease. Apo-B containing lipoproteins, including VLDL, also increase in nephrotic syndrome, which is caused by overproduction by the liver that concurrently increases albumin synthesis to compensate for renal protein wasting. Lipolysis disturbance can be reflected by elevated VLDL in uremia condition. During the third trimester of pregnancy, plasma triglyceride levels normally rise as much as threefold, but usually, this has little clinical consequences on its own. Some medications may increase plasma triglyceride levels, such as estrogens, glucocorticoids, some beta-blockers, tacrolimus, cyclosporine and thiazide diuretics. $(6,7)$

\section{Pathophysiology of Hypertriglyceridemia}

HTG can be induced by 1) disturbance of hepatic VLDL production and intestinal chylomicron production; 2) abnormalities of LPL-mediated lipolysis; or 3) dysfunctional remnant clearance. In intestinal chylomicron synthesis, dietary lipids are taken up in the enterocytes, incorporated into chylomicron particles that are secreted into the lymphatic system, whereas hepatic VLDL synthesis involved a series of intracellular process enabling the formation of lipid-enriched apo-B100 particles that can be excreted. LPL induce lipolysis of triglyceride in small tissues capillaries that need fatty acids for storage (adipose tissue) or energy (heart and skeletal muscle). glycosylphosphatidylinositol anchored high-density high-density lipoprotein 1 (GPIHBP1) is involved in the transport of LPL through endothelial cell layer to the cell surface, and then GPIHBP1 forms the platform to allow triglyceride hydrolysis Hepatic TRL clearance is a complicated process that involves three different receptors: LDL receptor (LDLr), LDLr-related protein 1 (LRP1), and heparansulfate proteoglycans (HSPG). Abnormalities in HSPG results in a mild hypertriglyceridemic phenotype due to delayed clearance of remnant particles.(8)

\section{Serum Triglycerides as Risk Factor for Cardiovascular Disease}

Triglycerides have routinely been identified as a 'risk factor' in case-control studies, even after adjustment for total cholesterol (TC) or HDL-C. There is some evidence that the level of triglyceride concentration may be even more predictive in women than in men.

Several clinical studies have been performed since 1990s, such as Copenhagen City Heart Study in 1994, a prospective observational survey that shows plasma triglycerides concentrations was significantly positively associated with risk of non-hemorrhagic events. The relative risk corresponding to an increase of this study of $1 \mathrm{mmol} / \mathrm{L}$ was 1.12 (95\% CI, 1.07-1.16). One of the limitations of this study is that no information of HDL-C was available, so that the data could not be adjusted for HDL-C.

Other studies were done in the following years. In 2009, Europe and North America assessments of the emerging risk factors collaborations evaluated 302,430 people free of know, vascular disease at baseline in 68 prospective studies. The hazard ratio (HR) for CVD with triglyceride was 1.37 (95\% CI, 1.31-1.42) after adjustment for non-lipid risk factors, but it was reduced to $0.99(95 \%$ CI, 0.94-1.05) after further adjustment for HDL-C and nonHDL-C.

Another the Copenhagen City Heart Study in 2011 demonstrated that stepwise increasing levels of nonfasting cholesterol and nonfasting triglycerides were similarly associated with stepwise increasing risk of myocardial infarction, with nonfasting triglyceride being the best predictor in women and non fasting cholesterol the best predictor in men.(6) Triglycerides can be degraded by most cells, neither can cholesterol. The cholesterol content of TRL is one of 
causes of atherosclerosis and CVD. Cholesterol accumulates in intimal foam cells and in atherosclerotic plaques, and remnant lipoproteins just like LDL can enter arterial intima, but chylomicrons are too large to enter. The atherogenicity of TRLs relates to the smaller size remnant particles that accumulate in plasma following lipolysis of chylomicrons and VLDL. Remnants, which are enriched in cholesterol and apo-E, can be taken up directly by macrophages leading to foam cell formation, while triglycerides could act through triglyceride hydrolysis. TRL remnants further contribute to atherosclerosis by impairing endothelial function, as well as by activating monocytes and inflammatory signaling pathways. Thrombogenicity and generation of thrombin can be stimulated by TRL and it also potentially inhibits fibrinolysis. Since remnant particles not only contain triglycerides but also approximately 40 times higher level of cholesteryl esters per particle compared with LDL, elevated levels of remnants may cause accelerated atherosclerosis and CVD. $(9,10,11)$

Very high triglyceride levels $(\geq 500 \mathrm{mg} / \mathrm{dL})$ correspondent to severe HTG, and very severe HTG $(\geq 2$,FGPIH $\mathrm{mg} / \mathrm{dL})$ increase the risk of acute pancreatitis, whereas mild or moderate HTG may be a risk factor for CVD. Other complications of very high triglyceride levels include skin manifestations (e.g., eruptive xanthoma) and fatty liver. Elevated triglyceride $\geq 200 \mathrm{mg} / \mathrm{mL}$ is associated with an increase risk for coronary heart disease (CHD). The result of 29 prospective studies analysis showed that patients in the highest versus the lowest tertile of triglyceride had an odds ratio of 1.72 for CHD (95\% CI, 1.56-1.90).(7,12)

In patients with very high triglyceride levels and patients with familial chylomicronemia syndrome the risk for atherosclerosis is attenuated, maybe since their plasma lipoprotein particles are too large to enter into the arterial intima. Nevertheless, there are many indications that there is an increased risk of $\mathrm{CV}$ disease in the marked or severe HTG as well.

Prospective studies have indicated that compared with fasting levels, non-fasting serum trygliceride levels may be a better or similar predictor of $\mathrm{CV}$ in the general population. In some of studies using standardized meals, greater $\mathrm{CV}$ risk was found to have associations with an increased HTG. The serum triglyceride concentration is often more strongly correlated with future coronary artery disease (CAD) incidence than in univariate analysis than is serum cholesterol. However, the strength of the apparent independent relationship between triglyceride and CAD is often weakened to the point of insignificance in individual trials in multiple logistic regression analysis, particularly when HDL-C is included. Raised triglyceride concentrations are strongly associated with low concentrations of HDL-C. Thus low HDL concentrations might merely be a long-term marker of raised TG and remnant cholesterol. Alternatively, HDL-C might be a marker of cardiovascular health but is non-causal in the atherogenesis.(12)

\section{Management of Hypertriglyceridemia}

The National Cholesterol Education Program Adult Treatment Panel (NCEP ATP) III recommends a non-HDL-C treatment goal as a secondary treatment target for patients with triglyceride $\geq 200 \mathrm{mg} / \mathrm{dL}$ with the primary treatment

Table 2. NCEP ATP III LDL-C and non-HDL-C treatment goals.(7) (with modifications)

\begin{tabular}{|c|c|c|}
\hline Risk category & LDL-C therapy goal & $\begin{array}{l}\text { Non-HDL-C therapy goal if } \\
\text { triglycerides } \geq 200 \mathrm{mg} / \mathrm{dL}\end{array}$ \\
\hline $\begin{array}{l}\text { High risk with } \mathrm{CHD} \text { or other equivalents } \\
(10 \text { year risk }>20 \%)\end{array}$ & $\begin{array}{c}<100 \mathrm{mg} / \mathrm{dL}(<70 \mathrm{mg} / \mathrm{dL} \text { as one of } \\
\text { targeted treatment })\end{array}$ & $\begin{array}{c}<130 \mathrm{mg} / \mathrm{dL}(<100 \mathrm{mg} / \mathrm{dL} \text { as one of } \\
\text { targeted treatment })\end{array}$ \\
\hline $\begin{array}{l}\text { Moderately high risk: }>2 \text { risk factors (10 } \\
\text { year risk } 10-20 \% \text { ) }\end{array}$ & $\begin{array}{c}<130 \mathrm{mg} / \mathrm{dL}(<100 \mathrm{mg} / \mathrm{dL} \text { as one of } \\
\text { targeted treatment })\end{array}$ & $\begin{array}{c}<160 \mathrm{mg} / \mathrm{dL}(<130 \mathrm{mg} / \mathrm{dL} \text { as one of } \\
\text { targeted treatment })\end{array}$ \\
\hline $\begin{array}{l}\text { Moderate risk: } 2 \text { risk factors ( } 10 \text { year } \\
\text { risk }<10 \% \text { ) }\end{array}$ & $<130 \mathrm{mg} / \mathrm{dL}$ & $<160 \mathrm{mg} / \mathrm{dL}$ \\
\hline Lower risk: 0 - 1 risk factor & $<160 \mathrm{mg} / \mathrm{dL}$ & $<190 \mathrm{mg} / \mathrm{dL}$ \\
\hline
\end{tabular}


target being LDL-C (Table 2). Non-HDL-C, calculated as TC - HDL-C, involves cholesterol carried by atherogenic lipoprotein: LDL, lipoprotein (a), IDL, VLDL, chylomicron and their remnants. Compared with LDL-C, non-HDL-C correlates more strongly with the apo-B concentration. Apo-B level is common considered an indicator of number of circulating atherogenic lipoprotein particles due to apo-B involvement on atherogenic lipoprotein particle.(7)

Mild-to-moderate HTG can be primarily managed by lifestyle therapy, including appropriate diet composition, physical activity and a program to achieve weight reduction. The primary goal of therapy in severe HTG is to lower triglyceride levels to prevent pancreatitis.

Lately studies have focused on the type carbohydrate that may affect serum triglyceride levels, and in particular on the role of fructose. Moderating intakes of foods or beverages with high fructose content and/or high glycemic index may help to lower triglyceride concentration. Smoking cessation and reducing or elimitaing alcohol consumption also often lower elevated triglyceride levels.

Pharmacological therapy includes statins, fibrates, n-3 fatty acids, and niacin. Those all substantially lower triglyceride. The best reasonable approach is to lower triglycerides levels, fibrates, and n-3 fatty acids. On the other hand, if the primary goal is to modify the size and density of LDL and HDL particles, niacin might be best. Lipoprotein (a) levels, an independent risk factor for CVD, also can be reduced by niacin. Due to niacin effect on elevated cholesterol levels and HTG, many patients will already be on statins; a hyperglycemic drug might than be added to a statin-based treatment. $(7,13)$

In combination therapy of fenofibrate add-on to statin, lipid effects associated with fenofibrate use include reduced triglyceride levels, increased HDL-C levels and a reduction in the number of small, dense LDL particles, which arise due to changes in the expression of genes that modify lipid metabolism. $(2,13)$

Several large-scaled studies have provided clinical benefits of fenobrate-statin combination therapy. The recent one is a meta-analysis of five placebo-controlled studies (Action to Control Cardiovascular Risk in Diabetes (ACCORD), Fenofibrate Intercention and Event Lowering in Diabetes (FIELD), Bezafibrate Inferction Prevention (BIP), Helsinki Heart Study (HHS) and Veterans Affairs HDL Intervention Trial (VA-HIT)) on a subgroup of 4726 subjects with $\mathrm{AD}$, which found that fibrate use was associated with a $35 \%$ reduction in the risk of coronary events. $(2,7)$
Fenofibrate has been approved by The European Medicines Agency as an adjunct to improving diet and other non-pharmacological therapies (e.g., exercise, weight reduction) for the treatment of: 1) severe HTG, with or without low levels of HDL-C; 2) mixed hyperlipidemia when a statin is contra-indicated or not tolerated; and 3) mixed hyperlipidemia in high CVD risk patients, in addition to a statin when triglycerides and HDL-C levels are poorly controlled. It has also been used for a sufficiently long period of time, and there is no increased incidence of adverse events or serious adverse events compared with either monotherapy. $(2,11)$

\section{Conclusion}

HTG is common and present in about a third of the population. In a recent meta-analysis, triglycerides levels have been associated with cardiovascular events but not with the mortality. The majority of hypertriglyceridemic patients have mild or moderate triglyceride levels $(<1,000$ $\mathrm{mg} / \mathrm{dL}$ ) and treatment should be focused on reduction of cardiovascular risk. Patients with severe or very severe HTG $(>1,000 \mathrm{mg} / \mathrm{dL})$ are at risk for pancreatitis. Although results are suggestive of an association between non-fasting triglyceride levels and cardiovascular risk, diagnosis of HTG is best based on fasting levels until standardized sampling conditions are established.

Since HTG has been established as an important risk factor for CVD, the condition should be treated. Non-HDL-C levels, as defined by NCEP-ATP III guidelines, should be considered as a treatment goal in patients with moderate HTG. Treatment for HTG should be initiated with lifestyle therapy, while addition of hypotriglyceridemic drugs may be considered if patients do not reach treatment goals. In patients with severe or very severe HTG, a fibrate should be used as a first-line agent, while in patients with moderate HTG, a statin-based combination therapy is more common.

\section{References}

1. Chan DC, Barrett PHR, Watts GF. The metabolic and pharmacologic bases for treating atherogenic dyslipidemia. Best Pract Res Clin Endocrinol Metab. 2014; 28: 369-85. 
2. Aguiar C, Alegria E, Bonadonna RC, Catapano AL, Consentino $\mathrm{F}$, Elisaf $\mathrm{M}$, et al. A review of the evidence on reducing macrovascular risk in patients with atherogenic dyslipidemia: A report from an expert consensus meeting on the role of fenofibrate-statin combination therapy. Atheroclerosis. 2015; 19 (Suppl): 1-12.

3. Sparks JD, Sparks CE, Adeli K. Selective hepatic insulin resistance, VLDL overproduction, and hypertriglyceridemia. Arterioscler Throm Vasc Biol. 2012; 32: 2104-12.

4. Yuan G, Al-Shali KZ, Hegele RA. Hypertriglyceridemia: Its etiology, effects and treatment. Can Med Assoc J. 2007; 176: 1113-20.

5. Balakumar P, Babbar L, Kalra S, Mahadevan N, Sritharan S, Krishan P. Is hypertriglyceridemia a key detrimental factor or associative triggering factor for cardiovascular abnormalities? Syst Rev Pharm. 2012; 3: 1-3.

6. Boullart ACI, de Graaf J, Stalenhoef AF. Serum triglycerides and risk of cardiovascular disease. Biochimica Biophysica Acta. 2012; 1821: 867-75.

7. Maki KC, Bays HE, Dicklin MR. Treatment options for the management of hypertriglyceridemia: Strategies based on the best-available evidence. J Clin Lipidol. 2012; 6: 413-26.

8. Hassing HC, Surendran RP, Mooij HL, Stroed ES, Nieuwdorp M, Dallinga-Thie GM. Pathophysiology of hypertriglyceridemia. Biochimica Biophysica Acta. 2012; 1821: 826-32.

9. Nordestgaard BG, Varbo A. Triglycerides and cardiovascular disease. Lancet. 2014; 384: 626-35.

10. Borén J, Matikainen N, Adiels M, Taskinen MR. Postprandial hypertriglyceridemia as a coronary risk factor. Clinica Chimica Acta. 2014; 431: 131-42.

11. Watts GF, Karpe F. Why, when and how should hypertriglyceridemia be treated in the high-risk cardiovascular patient? Expert Rev Cardiovasc Ther. 2011; 9: 987-97.

12. Tenenbaum A, Klempfner R, Fisman EZ. Hypertriglyceridemia: A too long unfairly neglected major cardiovascular risk factor. Cardiovasc Diabetol. 2014; 13: 159-68.

13. Berglund L, Brunzell JD, Goldberg AC, Goldberg IJ, Stalenhoef A. Treatment options for hypertriglyceridemia: From risk reduction to pancreatitis. Best Pract Res Clin Endocrinol Metab. 2014; 28: 423-37. 